\title{
APLICAÇÃO DA ESCALA DE AVALIAÇÃO DO USO DE FRALDAS EM UMA CLIINICA MÉDICA DE UM HOSPITAL UNIVERSITÁRIO
}

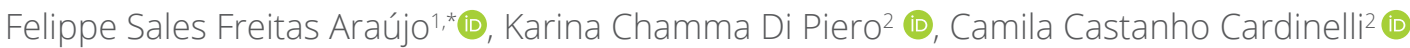

\section{RESUMO}

Objetivos: aplicar a escala de avaliação do uso fraldas e absorventes (AUFA) nos pacientes internados na clínica médica; identificar e analisar os resultados dessa aplicação, o perfil sociodemográfico dos pacientes em vogo bem como repercussões à pele relacionadas ao uso de absorventes e fraldas. Método: estudo observacional transversal, descritivo e exploratório, realizado no setor de clínica médica do Hospital Universitário Clementino Fraga Filho (HUCFF/UFRJ), no período de novembro de 2019 a fevereiro de 2020, com amostra por conveniência de 46 pacientes. Ocorreu aplicação de ficha para levantamento de dados gerais e, após, a AUFA, que identificou as seguintes variáveis: condições da pele, envelhecimento da pele, capacidade cognitiva, capacidade motora e incontinências. Resultados: todos os 46 pacientes encontravam-se em uso de fralda, sendo metade mulheres e a outra metade homens, em grande maioria composta de idosos e sem diagnóstico de incontinência. Diante da aplicação e análise da AUFA, apenas 18 pacientes $(39,14 \%)$ tinham indicação para uso de fraldas. Com o uso inadequado da fralda, 27 pacientes apresentaram lesão de pele decorrente de umidade, situação que se justificou pela ausência da observação de cuidados sistematizados para prevenção e tratamento de dermatite associada à incontinência nos pacientes avaliados. Conclusão: indica-se o uso da escala AUFA para identificar os pacientes que necessitam usar fralda, delimitando cuidados com seu manejo e prevenindo, assim, complicações e agravamentos da incontinência.

DESCRITORES: Enfermagem. Cuidados de enfermagem. Incontinência urinária. Absorventes higiênicos. Prevenção de doenças. Estomaterapia.

\section{APPLICATION OF THE DIAPER USE RATING SCALE IN A MEDICAL CLINIC OF A UNIVERSITY HOSPITAL}

\begin{abstract}
Objectives: apply the evaluation scale for the use of diapers and absorbent products (AUFA Scale) in patients admitted to the medical clinic; identify and analyze the results of this application, the sociodemographic profile of patients in vogue as well as repercussions on the skin related to the use of sanitary pads and diapers. Method: observational, cross-sectional, descriptive and exploratory study, carried out in the medical clinic sector of the University Hospital Hospital Universitário Clementino Fraga Filho (HUCFF/UFRJ), from November 2019 to February 2020, with a convenience sample of 46 patients. There was application of a form to survey general data and, later, the application of the AUFA scale, which identified the following variables: skin conditions, skin aging, cognitive
\end{abstract}

1. Universidade Federal do Rio de Janeiro - Hospital Universitário Clementino Fraga Filho - Residência Multiprofissional em Clínica Médica - Rio de Janeiro (RJ), Brasil.

2. Universidade Federal do Rio de Janeiro - Hospital Universitário Clementino Fraga Filho - Comissão de Métodos Relacionados à Integridade da Pele - Rio de Janeiro (RJ), Brasil.

*Autor correspondente: felippesales-@hotmail.com

Editor de Seção: Gisela Maria Assis

Recebido: Jul. 2021, 23 | Aceito: Set. 2021, 15

Como citar: Araújo FSF; Di Piero KC; Cardinelli CC. Aplicação da escala de avaliação do uso de fraldas em uma clínica médica de um hospital universitário. ESTIMA, Braz. J. Enterostomal Ther., 2021, 19: e2221. https://doi.org/10.30886/estima.v19.1114_PT 
ability, motor skills and incontinence. Results: all 46 patients were using diapers, half women and the other half men, mostly elderly and without a diagnosis of incontinence. In view of the application and analysis of the AUFA scale, only 18 patients (39.14\%) were indicated for diaper use. With the inappropriate use of diapers, 27 patients had skin lesions resulting from moisture, a situation that was justified by the absence of systematic care observation for the prevention and treatment of dermatitis associated with incontinence in the evaluated patients. Conclusion: the use of the AUFA scale is indicated to identify patients who need to wear diapers, delimiting care with their management and thus preventing complications and worsening of incontinence.

DESCRIPTORS: Nursing. Nursing care. Urinary incontinence. Sanitary pads. Prevention of diseases. Stomatherapy.

\section{APLICACIÓN DE LA ESCALA DE EVALUACIÓN DEL USO DE PAÑALES EN UNA CLÍNICA MÉDICA DE UN HOSPITAL UNIVERSITARIO}

\section{RESUMEN}

Objetivos: aplicar la escala de evaluación del uso pañales y toallas sanitarias (AUFA) en los pacientes internados en la clínica médica; identificar y analizar los resultados de esta aplicación, el perfil sociodemográfico de los pacientes en estudio, así como repercusiones para la piel relacionadas al uso de toallas sanitarias y pañales. Método: estudio observacional transversal, descriptivo y exploratorio, realizado en el sector de clínica médica del Hospital Universitario Clementino Fraga Filho (HUCFF/UFRJ), en el período de noviembre del 2019 a febrero del 2020, con muestra por conveniencia de 46 pacientes. Ocurrió la aplicación de ficha para el levantamiento de datos generales y, después, la AUFA, que identificó las siguientes variables: condiciones de la piel, envejecimiento de la piel, capacidad cognitiva, capacidad motora e incontinencias. Resultados: los 46 pacientes se encontraban en uso de pañal, siendo mitad mujeres y la otra mitad hombres, en gran mayoría compuesta por ancianos y sin diagnóstico de incontinencia. Ante la aplicación y análisis de la AUFA, solamente 18 pacientes $(39,14 \%)$ tenían indicación para el uso de pañales. Con el uso inadecuado del pañal, 27 pacientes presentaron lesión de piel derivada de humedad, situación que se justificó por la ausencia de la observación de cuidados sistematizados para prevención y tratamiento de dermatitis asociada a la incontinencia en los pacientes evaluados. Conclusión: se indica el uso de la escala AUFA para identificar a los pacientes que necesitan usar pañal, delimitando cuidados con su manejo y previniendo, así, complicaciones y agravamientos de la incontinencia.

DESCRIPTORES: Enfermería. Cuidados de enfermería. Incontinencia urinaria. Toallas sanitarias higiénicas. Prevención de enfermedades. Estomaterapia.

\section{INTRODUÇÃO}

O presente estudo possui como objeto as ações de enfermagem relacionadas ao manejo da incontinência em relação ao uso de dispositivos absorventes e fraldas, além da prevenção da dermatite associada à incontinência em pacientes internados no setor de clínica médica de um hospital universitário localizado no município do Rio de Janeiro.

Os dispositivos absorventes para uso adulto são considerados pela literatura científica como ferramentas agregadas ao cuidado em saúde. Nessa perspectiva, sua incorporação na assistência à saúde demanda o julgamento clínico e reavaliação constante das práticas de cuidado.

O enfermeiro precisa fundamentar-se e instrumentalizar-se na indicação adequada e continuidade quanto ao uso na prática clínica desses dispositivos, consciente de que essa prática acarreta benefícios e prejuízos, como a dermatite associada à incontinência (DAI), lesão por pressão, infecção urinária, piora da incontinência, dentre outros ${ }^{1-6}$.

Nesse sentido, faz-se necessário a utilização de escalas e instrumentos de avaliação para subsidiar a tomada de decisão consciente e embasada em evidências científicas pelo enfermeiro quanto ao uso de absorventes e fraldas ${ }^{1-6}$.

Assim, a escala de avaliação do uso fraldas e absorventes (AUFA) mostra-se adequada por ser uma ferramenta sistematizada para indicação e avaliação da necessidade do uso de dispositivos absorventes, bem como auxiliadora na implementação de medidas preventivas de DAI, conforme determinação de escore de risco ao paciente ${ }^{1-6}$. 
Ademais, ao aplicar a referida escala encontra-se um escore com algorítimo que auxilia e embasa a escolha do dispositivo absorvente adequado. Assim, se a pontuação final for menor que 11 não há indicação para o uso de dispositivos absorventes, e sugere-se a utilização de utensílio externo, como comadre, patinho ou vaso sanitário. Nesse caso, a perda urinária ou fecal está ausente ou é menor que $50 \mathrm{~mL}$ ao dia, associada à capacidade cognitiva preservada ${ }^{1}$.

Entretanto, se o resultado for maior ou igual a 11 pontos e menor que 14 pontos, indica-se que há perda urinária ou fecal entre 50 a $900 \mathrm{~mL}$ ao dia, correlacionado ao déficit cognitivo parcial, logo, há necessidade do uso de absorventes ${ }^{1}$.

Por fim, se for alcançado o quociente maior ou igual a 14 pontos na escala AUFA, revela-se que há perda urinária ou fecal maior ou igual a $900 \mathrm{~mL}$ por dia, com déficit cognitivo grave, dessa forma, recomenda-se a utilização de fraldas ${ }^{1}$.

Portanto, a escala AUFA norteia a tomada de decisão do enfermeiro, possibilitando o planejamento do cuidado sistematizado e individualizado, sendo os principais elementos analisados: a capacidade cognitiva e motora, grau de incontinência, preferências do paciente e cuidador, número de trocas e condições da pele - envelhecimento e integridade da pele ${ }^{1-6}$.

Vale lembrar que a quebra da integridade da pele representa um problema de saúde que pode interferir diretamente na qualidade de vida das pessoas, capacidade funcional, autonomia e independência. Quando associada a quaisquer doenças crônicas amplia ainda mais a vulnerabilidade quanto aos processos patológicos infecciosos que podem culminar com hospitalização. Dessa forma, a prevenção é a melhor alternativa, cabendo aos profissionais de saúde, especialmente ao enfermeiro, intervenções preventivas e tratamento precoce das lesões associadas ${ }^{7}$.

É relevante ressaltar que nos últimos anos, com os avanços tecnológicos em saúde, observou-se um aumento significativo na expectativa de vida da população mundial, e, portanto, mudança no perfil dos pacientes sob cuidados de saúde, com idades mais avançadas, acometidos de doenças crônicas e risco para lesões de pele ${ }^{6-8}$.

No que se refere à incontinência urinária, fecal e dupla (urinária e fecal), o uso de fraldas ou absorventes podem ser considerados responsáveis pelo surgimento de agravos dermatológicos e ampliação dos episódios de incontinência. Dessa forma, a indicação para tal uso deve ser avaliada, individualmente, pelo enfermeiro mediante estratégias científicas. A escolha do dispositivo absorvente deve ocorrer conforme análise das características do paciente, como peso e altura, bem como tipo de incontinência ${ }^{9}$.

Logo, tipo, formato, capacidade e velocidade de absorção do absorvente ou fralda é o período de saturação, inibidores de odores e as necessidades de trocas são pontos a serem considerados na escolha do dispositivo, conforme demandada do perfil da clientela ${ }^{9}$.

Entretanto, na prática cotidiana, o uso dessa avaliação individual não é rotineiro nas unidades de internação. Não é possível determinar se os parâmetros designados pelos fabricantes como indicação de uso para cada subgrupo estão em consonância com os parâmetros técnicos da Sociedade Internacional de Continência (ICS). Portanto, o uso e indicação empírica podem trazer consequências negativas para o paciente e elevado custo para a instituição de saúde9 .

Nesse sentido, delimitou-se como questão norteadora a seguinte pergunta: como ocorre a indicação do uso de fraldas e absorventes pela aplicação da escala AUFA aos pacientes internados no setor de clínica médica de um hospital universitário do Rio de Janeiro?

Para responder à questão supracitada foram delimitados os seguintes objetivos: aplicar a escala AUFA nos pacientes internados na clínica médica; identificar e analisar os resultados dessa aplicação, o perfil sociodemográfico dos pacientes em vogo bem como repercussões à pele relacionadas ao uso de absorventes e fraldas.

\section{MÉTODO}

Tratar-se de um estudo observacional transversal, descritivo e exploratório, realizado no Hospital Universitário Clementino Fraga Filho (HUCFF) da Universidade Federal do Rio de Janeiro (UFRJ).

O método utilizado foi observacional, com delineamento transversal, sendo determinado um intervalo de tempo para condução da pesquisa, possibilitando um retrato da realidade momentânea ${ }^{10}$.

O caráter descritivo e exploratório descreve características da população,fenômenos, relações de experiências mediante observações, registros, análises, classificações e compreensões dos atos, sem interferir na realidade analisada, esclarecendo conceitos e ideias ${ }^{11}$. 
O cenário do estudo foi o setor de clínica médica do HUCFF/UFRJ, localizado no município do Rio de Janeiro. Tal setor possui 46 leitos, divididos em 2 enfermarias, uma feminina e uma masculina com o mesmo quantitativo de leitos.

Os participantes foram os pacientes internados na unidade de clínica médica do referido hospital. Esse estudo abordou a totalidade de pacientes internados no período da coleta de dados. Assim, foram 46 participantes, de ambos os sexos e com idade igual ou superior a 18 anos, que durante o período de coleta de dados aceitaram por livre escolha participar da pesquisa e assinaram o termo de consentimento livre e esclarecido.

Todos os pacientes que participaram do estudo tiveram assegurados os preceitos legais e éticos, com base na aprovação do Comitê de Ética em Pesquisa do HUCFF/UFRJ, CAAE no 31521220. 4.0000.5257 e número do parecer 4.268.134.

A coleta de dados ocorreu entre os meses de novembro de 2019 e fevereiro de 2020 por meio da aplicação de uma ficha para levantamento de dados gerais contendo: sexo, idade, raça, doenças de base e motivo da internação atual.

Após a identificação da amostra, aplicou-se a Escala_AUFA ${ }^{\circledR}$.Complementarmente, aplicaram-se a Escala Mini Exame Do Estado Mental (MEEM) e International Consultation on Incontinence Questionnaire/Short FORM (ICIQ-SF), ambos para confirmar situações relacionadas à cognição e incontinências identificadas.

Sobre a escala AUFA, clarifica-se que essa emergiu como produto de uma pesquisa de mestrado, sendo atualizada e validada por uma pesquisa de doutorado em enfermagem ${ }^{1}$.

A escala AUFA avalia as preferências do paciente/cuidador, número de trocas, integridade da pele, envelhecimento da pele, capacidade cognitiva, capacidade motora e incontinência, com escores entre 1 e 3 para cada item, sendo o resultado do escore final a soma dos pontos: $<11$ pontos - sem indicação no uso de absorventes ou fraldas; $\geq 11$ e $<14$ pontos indicação de uso de absorventes; $\geq 14$ pontos - indicação de uso de fraldas; < 17 pontos - risco menor de DAI; $\geq 17$ pontos - risco maior de $\mathrm{DAI}^{1}$ (Fig. 1).

Em caráter complementar foram utilizados os instrumentos MEEM (Fig. 2) e ICIQ-SF (Fig. 3) a seguir:

$\mathrm{O}$ tratamento e análise dos dados se deram em duas partes: os elementos provenientes da primeira fase do instrumento de coleta dos dados, que consiste na ficha para levantamento de dados gerais do participante da pesquisa foram incluídas e analisadas no software Microsoft Excel ${ }^{\circledR}$ 2007, dando origem aos dados de identificação da população.

Comparando os dados da aplicação da Escala_AUFA ${ }^{\circledR}$, construiu-se tabelas no Word@ para analisar e comparar escores e dados complementares com base no MEEM e ICIQ-SF.

\section{RESULTADOS}

Dos 46 participantes analisados, 23 (50\%) pacientes foram do sexo masculino e 23 (50\%) do feminino. As idades variaram entre 40 e 99 anos, com maior prevalência na faixa etária de 60 a 69 anos. Vinte e um participantes (45,65\%) declararam-se brancos e 25 (54,35\%) negros.

Sobre o grau de escolaridade, todos possuíam fundamental incompleto. Quanto ao motivo da internação, todos apresentavam hipertensão arterial sistêmica, sendo a grande maioria internada por acidente vascular cerebral, seguido de infarto agudo do miocárdio e dispneia, conforme tabela a seguir de caracterização da população estudada (Tabela 1).

Ao aplicar a escala AUFA, observou-se que $100 \%$ dos participantes utilizavam fralda, sendo que 28 (60,86\%) não apresentavam indicação para o uso de dispositivo absorvente e $18(39,14 \%)$ apresentavam escore compatível com utilização de fraldas ou dispositivos absorventes.

Diante disso, observou-se uma tendência de utilização da fralda sem julgamento científico prévio pela enfermagem, inclusive com interferência da decisão pelo paciente, sua família e/ou cuidador, que também não apresentavam justificativas científicas para seu uso.

Foi avaliada a capacidade cognitiva dos pacientes pela escala MEEM, sendo $21(45,65 \%)$ com escore preservado, 12 $(26,09 \%)$ com queixas subjetivas de memória e 13 (28,26\%) com queixas objetivas de memória. Isso significou um aumento de risco em mais da metade dos participantes sobre a compreensão da necessidade do uso da fralda e seu adequado uso na prática clínica. 


\begin{tabular}{|c|c|}
\hline FATORES & AVALIAÇÃO \\
\hline \multirow{3}{*}{ Preferências do paciente/cuidador } & 1 Dispositivos externos (vaso sanitário, comadre, patinho, cateter com preservativo) \\
\hline & 2 Uso de absorventes \\
\hline & 3 Uso de fraldas geriátricas \\
\hline \multirow{3}{*}{ Condições da pele } & 1 Íntegra \\
\hline & 2 Hiperemia em genitália, glúteo, região inguinal e/ou abdômen inferior \\
\hline & 3 Lesão ulcerada em genitália, glúteo, região inguinal e/ou abdômen inferior \\
\hline \multirow{3}{*}{ Envelhecimento da pele } & 1 Elasticidade preservada \\
\hline & 2 Prova de turgor $\geq 2$ e $<5$ segundos \\
\hline & 3 Prova de turgor $\geq 5$ segundos \\
\hline \multirow{3}{*}{ Capacidade cognitiva } & 1 Preservada \\
\hline & 2 Queixa subjetiva de memória \\
\hline & 3 Queixa objetiva de memória \\
\hline \multirow{3}{*}{ Capacidade motora } & 1 Independente para atividades de vida diária \\
\hline & 2 Parcialmente dependente para atividades de vida diária \\
\hline & 3 Dependente para atividades de vida diária \\
\hline \multirow{3}{*}{ Incontinência } & 1 Incontinência ausente \\
\hline & 2 Incontinência leve \\
\hline & 3 Incontinência moderada a grave \\
\hline
\end{tabular}

Algoritmo de decisão da Escala de Avaliação do uso de fraldas e absorventes para o controle urinário e fecal

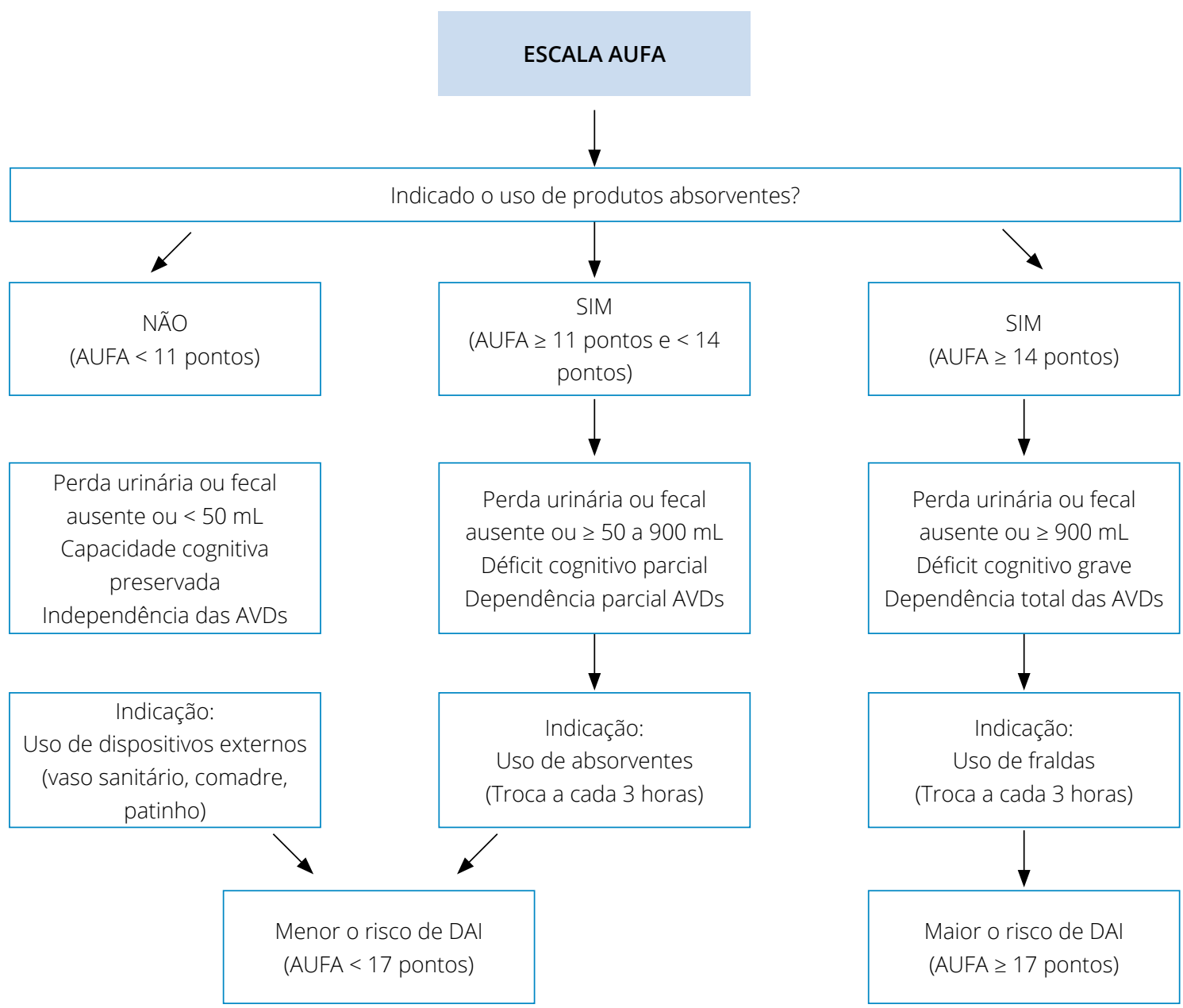

Figura 1. Instrumento de indicação do uso de absorventes ou fraldas e de avaliação do risco de dermatite associada à incontinência. Rio de janeiro (RJ) - 2019.

AUFA= Avaliação do Uso de Fraldas e Absorventes; AVD= Atividades de Vida Diária; DAl= Dermatite Associada à Incontinência. 


\section{ORIENTAÇÃO 01 PONTO PARA CADA}

1) Em que ano estamos?

2) Que horas são aproximadamente?

3) Em que dia do mês estamos?

4) Em que dia da semana estamos?

5) Em que mês estamos?

6) Onde estamos?

7) Em que andar estamos?

8) Em que bairro estamos?

9) Em que cidade estamos?

10) Em que estado estamos?

\section{REGISTRO 03 PONTOS}

11) Mencione 3 objetos (COPO, MALA e CARRO), e peça para repetir. Dê um ponto para cada resposta correta. Repita até que ele aprenda.

Número de tentativas:

(se houver "inclusão", descrever):

\section{ATENÇÃO E CÁLCULO 05 PONTOS}

12) Série de "7". Dê 100 peça para subtrair 7, repita 5 vezes $(93,86,79,72,65)$.

Ou Peça para soletrar a palavra mundo de trás para frente (O-D-N-U-M)

MEMÓRIA DE EVOCAÇÃO 03 PONTOS

13) Peça para repetir as 3 palavras. Retire 1 ponto para cada objeto não citado. Máximo de 6 tentativas

LINGUAGEM 09 PONTOS

14) Aponte o lápis e o relógio e peça para nomeá-los (02 pontos)

15) Peça par repetir: "nem aqui, nem ali, nem lá" (01 ponto)

16) Peça para seguir um comando em três etapas: "pegue este papel com a mão direita, dobre ao meio e ponha no chão" (03 pontos)

17) Peça para seguir o comando escrito no papel: "feche os olhos" (01 ponto)

18) Escreva uma sentença (deve ter sujeito, verbo e predicado) (01 ponto)

19) Peça para copiar o desenho (01 ponto)

TOTAL

Feche os olhos

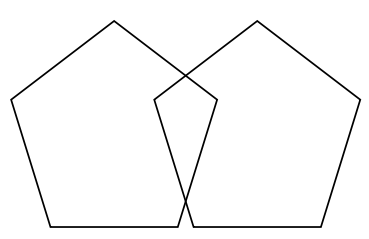

ANIMAIS

( ) Até 12 nomes diferentes

( ) Entre 13 e 18 nomes

( ) Mais de 18 nomes

Figura 2. Escala Mini Exame do Estado Mental: Avaliação Déficit Cognitivo. Rio de janeiro (RJ) - 2019. 


\section{ICIQ-SF EM PORTUGUÊS}

Nome do Paciente:

Data de Hoje

Muitas pessoas perdem urina alguma vez. Estamos tentando descobrir quantas pessoas perdem urina e o quanto isso as aborrece. Ficaríamos agradecidos se você pudesse nos responder as seguintes perguntas, pensando em como você tem passado, em média nas ÚLTIMAS QUATRO SEMANAS

1. Data de Nascimento_________ (Dia/Mês/Ano)=

2. Sexo: Feminino $\square$ Masculino $\square$

3. Com que frequência você perde urina? (assinale uma resposta)

$$
\begin{array}{r}
\text { Nunca } \square 0 \\
\text { Uma vez por semana ou menos } \square 1 \\
\text { Duas ou três vezes por semana } \square 2 \\
\text { Uma vez ao dia } \square 3 \\
\text { Diversas vezes ao dia } \square 4 \\
\text { O tempo todo } \square 5
\end{array}
$$

4. Gostaríamos de saber a quantidade de urina que você pensa que perde (assinale uma resposta) $=($

Nenhuma $\square 0$

Uma pequena quantidade $\square 2$ Uma moderada quantidade $\square 4$

Uma grande quantidade $\square 6$

5. Em geral, quanto que perder urina interfere em sua vida diária? Por favor, circule um número entre 0 (não interfere) e 10 (interfere muito)

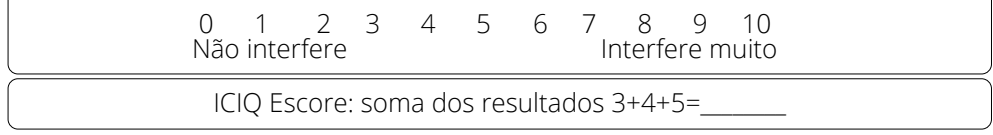

6. Quando você perde urina?

(por favor, assinale todas as alternativas que se aplicam à você)

Nunca $\square$ Perco quando tusso banheiro $\square$ Perco quando tusso ou espirro $\square$ Perco quando estou dormindo $\square$ Perco quando estou fazendo atividades físicas $\square$ Perco quando terminei de urinar e estou me vestindo $\square$ Perco sem razão óbvia $\square$ Perco o tempo todo $\square$

Figura 3. Escala International Consultation on Incontinence Questionnaire/ Short FORM. Rio de janeiro (RJ) - 2019.

Tabela1. Caracterização da amostra: sexo, idade, escolaridade, diagnóstico médico. Rio de Janeiro (RJ) - 2021.

\begin{tabular}{lcc}
\hline Sexo & Amostra & Raça/Cor \\
\hline Feminino & $23(50 \%)$ & 12 negras $(52,17 \%)$ \\
\hline Masculino & $23(50 \%)$ & 13 negros $(56,52 \%)$ \\
\hline Idade & & 10 brancos $(43,47 \%)$ \\
\hline $40-49$ anos & $02(4,35 \%)$ & \\
\hline $50-59$ anos & $09(19,56 \%)$ & $14(30,43 \%)$ \\
\hline $60-69$ anos & $12(26,09 \%)$ & $07(15,21 \%)$ \\
\hline $70-79$ anos & $02(4,35 \%)$ & \\
\hline $80-89$ anos & Escolaridade \\
\hline $90-99$ anos & Ensino Fundamental Incompleto (100\%) \\
\hline
\end{tabular}

continua... 
Tabela1. Continuação...

\begin{tabular}{|c|c|c|}
\hline Diagnóstico médico & Amostra & Patologia Comum \\
\hline Acidente vascular cerebral & $09(19,56 \%)$ & \multirow{20}{*}{ Hipertensão Arterial sistêmica (100\%) } \\
\hline Dispneia & $04(8,70 \%)$ & \\
\hline Infarto agudo do miocárdio & $04(8,70 \%)$ & \\
\hline Hemorragia digestiva & $03(6,52 \%)$ & \\
\hline Náuseas e vômitos & $03(6,52 \%)$ & \\
\hline Desnutrição & $03(6,52 \%)$ & \\
\hline Sepse & $03(6,52 \%)$ & \\
\hline Pielonefrite & $02(4,35 \%)$ & \\
\hline Encefalopatia hepática & $02(4,35 \%)$ & \\
\hline Isquemia MIE & $02(4,35 \%)$ & \\
\hline Cefaléia & $02(4,35 \%)$ & \\
\hline Reabilitação & $01(2,17 \%)$ & \\
\hline Celulite & $01(2,17 \%)$ & \\
\hline Radioterapia paliativa & $01(2,17 \%)$ & \\
\hline Vasculite & $01(2,17 \%)$ & \\
\hline Sangramento uretral & $01(2,17 \%)$ & \\
\hline Pênfigo vulgar & $01(2,17 \%)$ & \\
\hline Sincope & $01(2,17 \%)$ & \\
\hline Anemia & $01(2,17 \%)$ & \\
\hline Melanoma nasal & $01(2,17 \%)$ & \\
\hline
\end{tabular}

MIE $=$ Membro inferior esquerdo

Sobre as atividades da vida diária, 11 (23,91\%) pacientes classificaram-se como independentes, 12 (26,09\%) parcialmente dependentes e 23 (50\%), a maioria, dependentes para desenvolver atividades da vida diária, situação que determinava a necessidade da presença de um cuidador, interferindo positivamente ou negativamente na introdução do dispositivo absorvente e seu manejo.

A incontinência foi avaliada com auxilio da escala ICIQ-SF e foram encontrados $30(65,21 \%)$ pacientes com ausência de incontinência, 12 (26,09\%) com incontinência leve e 4 (8,70 \%) com incontinência moderada e grave, conforme a tabela 2, a seguir:

Os dados da pesquisa mostraram que o prejuízo na integridade da pele não ocorreu em 19 (41,30\%) participantes, entretanto mais da metade apresentou algum dano, sendo 09 (19,56\%) DAI grau 1 em áreas de genitália, glúteos, regiões inguinais e abdômen inferior, e 18 (39,14\%) DAI grau 3.

Tal fato pode estar relacionado também à utilização de duas ou mais fraldas, situação em desalinho com as boas práticas em saúde, favorecendo aumento de risco para o desenvolvimento de DAI, lesão por pressão, infecção urinária, entre outros.

A DAI é uma manifestação clínica comum em pacientes com incontinências. Trata-se de uma inflamação de pele, caracterizada por edema, eritema e lesões erosivas/maceradas da epiderme $e^{1-3,16}$ devido contato com excretas e umidade, especialmente nas regiões do períneo, genitália, glúteos, região perineal/perianal, coxas e parte superior do abdome/adjacências.

Os fatores que facilitam ou agravam a sua instalação compreendem desde o excesso da umidade da pele, cuidados inadequados no seu manejo, alterações do seu $\mathrm{pH}$ pela ação das excretas e/ou uso de produtos de higiene, além da presença de microrganismos e fatores extrínsecos como pressão e fricção ${ }^{3,16}$.

Diante disso, o manejo preventivo tem como objetivo a remoção de substâncias irritantes da pele, além do manuseio gentil, combinado com uso de barreiras protetoras de pele, ou seja, sistematização dos cuidados de enfermagem ${ }^{17}$. 
Tabela 2. Fatores das escalas: escala de avaliação do uso fraldas e absorventes, Escala Mini Exame do Estado Mental e International Consultation on Incontinence Questionnaire/Short FORM. Rio de Janeiro (RJ) - 2021.

\begin{tabular}{|c|c|c|c|}
\hline $\begin{array}{l}\text { Variáveis } \\
\text { ESCALA AUFA }\end{array}$ & Pontuação & Escore & Porcentagem \\
\hline \multirow{3}{*}{$\begin{array}{l}\text { Preferência do paciente/ } \\
\text { cuidado: }\end{array}$} & $\begin{array}{l}1 \text { Dispositivos externos (vaso sanitário, comadre, patinho, } \\
\text { cateter com preservativo) }\end{array}$ & 0 & $0 \%$ \\
\hline & 2 Uso de absorventes & 0 & $0 \%$ \\
\hline & 3 Uso de fraldas geriátricas & 46 & $100 \%$ \\
\hline \multirow{3}{*}{ Condições da pele } & 1 Íntegra & 19 & $41,30 \%$ \\
\hline & $\begin{array}{l}2 \text { Hiperemia em genitália, glúteo, região inguinal e/ou } \\
\text { abdômen inferior }\end{array}$ & 9 & $19,56 \%$ \\
\hline & $\begin{array}{l}3 \text { Lesão ulcerada em genitália, glúteo, região inguinal e/ou } \\
\text { abdômen inferior }\end{array}$ & 18 & $39,13 \%$ \\
\hline \multirow{3}{*}{ Envelhecimento da pele } & 1 Elasticidade preservada & 23 & $50 \%$ \\
\hline & 2 Prova de Turgor $\geq 2$ e $<5$ segundos & 21 & $45,65 \%$ \\
\hline & 3 Prova de turgor $\geq 5$ segundos & 2 & $4,34 \%$ \\
\hline \multirow{3}{*}{$\begin{array}{l}\text { Capacidade cognitiva- } \\
\text { MEEM (ensino fundamental } \\
\text { incompleto) }\end{array}$} & 1 Preservada & 21 & $45,65 \%$ \\
\hline & 2 Queixa subjetiva de memória & 12 & $26,09 \%$ \\
\hline & 3 Queixa objetiva de memória & 13 & $28,26 \%$ \\
\hline \multirow{3}{*}{ Capacidade motora } & 1 Independente para atividades de vida diária & 11 & $23,91 \%$ \\
\hline & 2 Parcialmente dependente para atividades de vida diária & 12 & $26,09 \%$ \\
\hline & 3 Dependente para atividades de vida diária & 23 & $50 \%$ \\
\hline \multirow{3}{*}{ Incontinência - ICIQ } & 1 Incontinência ausente & 30 & $65,21 \%$ \\
\hline & 2 Incontinência leve & 12 & $26,09 \%$ \\
\hline & 3 Incontinência moderada a grave & 4 & $8,70 \%$ \\
\hline
\end{tabular}

AUFA= Avaliação do Uso Fraldas e Absorventes; MEEM= Escala Mini Exame do Estado Mental; ICIQ=International Consultation on Incontinence Questionnaire/Short FORM

\section{DISCUSSÃO}

A análise do perfil dos participantes mostrou maioria de idosos, com frequência de idade acima de 60 anos, situação que reafirma o aumento dessa faixa etária, que pelo próprio declínio de suas funções fisiológicas já determina aumento de risco para complicações durante a internação, especialmente em um setor de clínica médica, com demandas diversas e diferentes pacientes internados ${ }^{18-20}$.

O processo de envelhecimento determina redução da capacidade funcional, suscetibilidade de doenças crônicas e da incontinência. Além disso, ainda tem a parcela cognitiva e nível de consciência que impactam na autonomia e autocuidado, exacerbando a vulnerabilidade e passividade do sujeito frente ao cuidador ${ }^{20}$.

Sobre a variável cor, a maioria dos participantes foi de negros, situação que suscita a importância de exames de pele precoces e mais minuciosos devida a complexidade na identificação de alterações iniciais, como eritema e lesões elementares difíceis de serem visualizadas na pele escura.

Sobre o grau de instrução, todos os participantes foram de nível fundamental, e sabendo-se que há relação direta entre escolaridade, qualidade de vida e saúde, observou-se que a baixa escolaridade/instrução dificultou a compreensão dos fatores que interferem na saúde individual.

Os motivos para internação atual dos participantes, bem como a doença de base comum entre eles estão inclusas no rol de doenças crônicas não transmissíveis, sendo a hipertensão arterial crônica prevalente, junto às complicações em destaque no estudo - acidente vascular cerebral e infarto agudo do miocárdio, assim como a dispneia enquanto sintoma que pode estar relacionado às complicações cardiopulmonares. 
Segundo Malta et al. ${ }^{21}$, portadores desse grupo de patologias usam duas vezes mais os serviços de saúde, com maior frequência e prevalência de internação e consultas médicas, impactando na qualidade de vida, saúde e gastos do sistema de saúde.

No que tange aos dados obtidos a partir da aplicação da escala AUFA, essa possibilitou a identificação da prática do uso de fraldas em adultos, especialmente em idosos no setor de clínica médica do hospital universitário, cenário do estudo, apontando para uma indicação sem critérios sistematizados preestabelecidos.

Ao indicar o uso de fraldas e dispositivos absorventes ao indivíduo de forma indiscriminada e sem uma avaliação criteriosa, assumem-se os riscos inerentes ao uso desse material, como a DAI, infecção urinária, lesão por pressão, dor e desconforto, e piora da incontinência urinária e/ou fecal. Além disso, dificulta-se o acompanhamento e manejo da incontinência ${ }^{22}$.

Por isso, esses indivíduos devem ser avaliados de forma integral, sistematizada com base em instrumentos científicos, tanto para a análise da saúde global e identificação do grau de complexidade do seu cuidado, quanto para critérios específicos relacionados à incontinência e necessidade de uso de dispositivo absorvente.

Além disso, o diagnóstico de incontinência deve ser prioridade no manejo desses pacientes com auxílio de especialistas, como o estomaterapeuta, para avaliar a indicação clínica, baseada em preceitos técnico-científicos, sobre a necessidade real do uso de dispositivos absorvente.

Dessa forma, evitando-se a implementação inadequada de fraldas e absorventes como uma rotina de organização do trabalho de enfermagem.

Diante disso, a prática do enfermeiro precisa ser fundamentada em preceitos teórico-práticos que respaldem a tomada de decisão em saúde e incorporação de materiais de higiene no cuidado. São imprescindíveis instrumentos que sistematizem a avaliação de critérios para o uso de fralda em pacientes adultos.

A aplicação da escala AUFA parece ser uma alternativa para indicação do uso de dispositivos absorventes ${ }^{6,22}$, entretanto, é necessária a discussão do critério disposto na escala relacionada à orientação do cuidador na sistematização de horários para a troca da fralda, especificamente de $3 \mathrm{em} 3$ horas, situação que pode determinar conflito conforme as características da incontinência individual ${ }^{1,6,22}$.

Porém sem estudos prospectivos de avaliação sobre periodicidade de troca de fralda, há uma lacuna científica quanto a esse consenso, situação que precisa ser individualizada em relação às variáveis intervenientes como: grau de incontinência, volume de micção, aspectos da pele, lesões pré-existentes, mobilidade, etc.

Além disso, cada fralda e absorvente possui um grau de absorção e saturação de acordo com as especificações do fabricante. Todos esses elementos devem ser levados em consideração para definir o momento de troca da fralda, portanto mais estudos são necessários para recomendações clínicas consensuais.

Desse modo, a ampla educação em saúde conforme boas práticas no manejo de absorventes e fraldas com base nos consensos clínicos em saúde auxilia a política institucional na qualidade e segurança do paciente.

Por fim, considera-se como limitação para este estudo a realização de critérios clínicos relacionados ao uso da fralda determinados pela escala AUFA em uma amostra pequena e de apenas um cenário assistencial em um hospital universitário. Apesar de ter-se abordado o quantitativo integral de pacientes internados na clínica médica no período da coleta de dados. Sobretudo, as contribuições foram satisfatórias e descortinaram uma lacuna na assistência que deve ser suprida.

Diante disso, recomenda-se mais estudos sobre a temática, além de custo-efetividade acerca do dispositivo absorvente fralda relacionado à sua aplicação clínica com maior número de participantes, tendo em vista boas práticas de utilização. Além da realização do mesmo estudo em outros cenários assistenciais, para avaliação da escala AUFA com vistas à tomada de decisão da indicação da fralda e seu manejo, inclusive o momento correto para a troca, com objetivo na prevenção de complicações relacionadas à pele.

\section{CONCLUSÃO}

Foi possível concluir com este estudo que há necessidade de implantar a sistematização da assistência em enfermagem para indicação e manejo do uso de absorvente e fralda em pacientes adultos, em geral idosos.

O uso inadequado ou sem indicação deve impactar negativamente nas condições de saúde, especialmente na pele desses pacientes e no custo da assistência em saúde durante a sua internação. 
A sistematização da assistência de enfermagem somada à utilização de escalas para avaliar e apoiar a tomada de decisão, como a escala AUFA, garantirá ao paciente com incontinência e utilização de absorvente ou fralda cuidados seguros e eficientes, que é a prática baseada em evidências científicas.

A fundamentação científica para a assistência é necessária e reforçada pela Política Nacional de Segurança do Paciente, que traça eixos para o delineamento de uma assistência segura em saúde. Ações voltadas para prevenção, promoção da saúde e uso seguro de tecnologias/materiais com base em conceitos técnico científicos ampara as decisões para a assistência.

O absorvente e a fralda são materiais de higiene agregados ao cuidado em saúde, logo seu uso deve ser justificado, sendo necessários protocolos visando à segurança do paciente, especialmente com análise do perfil do usuário, facilitado por instrumentos como a escala AUFA.

Diante disso, este estudo possibilitou identificar que a indicação e até mesmo manejo de absorvente e fralda pela enfermagem ainda não está sistematizada e baseada em ciência. Portanto, a utilização da escala AUFA se mostrou relevante cientificamente para guiar a análise individualizada da clientela.

\section{CONTRIBUIÇÃO DOS AUTORES}

Conceitualização: Araujo FSF e Di Piero KC; Metodologia: Di Piero KC; Investigação: Araujo FSF e Di Piero KC; Redação - Primeira versão: Araujo FSF, Di Piero KC e Cardinelli CC; Redação - Revisão \& Edição: Araujo FSF, Di Piero KC e Cardinelli CC; Supervisão: Di Piero KC.

\section{DISPONIBILIDADE DE DADOS DE PESQUISA}

Todos os dados foram gerados ou analisados no presente estudo.

\section{REFERÊNCIAS}

1. Bitencourt G R. Validação da escala de avaliação do uso de fraldas e absorventes em idosos na atenção primária [tese]. Rio de Janeiro (RJ): Escola de Enfermagem Aurora de Afonso Costa, Universidade Federal Fluminense; 2019.

2. Beeckman D, Van den Bussche K, Alves P, Beele H, Ciprandi G, Coyer F et al. The Ghent Global IAD Categorisation Tool (GLOBIAD). Skin Integrity Research Group - Ghent University. 2017. Disponível em: www.UCVVGent.be

3. Koudounas S, Bader DL, Voegeli D. Knowledge Gaps in the Etiology and Pathophysiology of Incontinence-Associated Dermatitis: A Scoping Review. J Wound Ostomy Continence Nurs. 2020; 47(4): 388-95. https://doi.org/10.1097/WON.0000000000000656

4. Fernández FPG, Casanova PL, Palma MR, Goméz TS, Agreda JJS. Guia: cuidados de la piel en pacientes con incontinência y prevención de lesiones asociadas de la humedad. Departamento de enfermeria comunitaria, medicina preventiva y salud pública e historia de la ciência; 2016. Disponível em: https://gneaupp.info/cuidados-de-la-piel-en-pacientes-con-incontinenciay-prevencion-de-lesiones-asociadas-a-la-humedad/

5. Cunha CV, Ferreira D, Nascimento D, Felix F, Cunha P, Penna LHG. Dermatite associada à incontinência em idosos: caracterização, prevenção e tratamento. ESTIMA, Braz J Enterestomal Ther. 2016; 13(3). Disponível em: https://www.revistaestima.com.br/ estima/article/view/218

6. Bitencourt GR, Santana RF. Evaluation scale for the use of adult diapers and absorbent products: methodological study. Online Braz J Nurs. 2021; 20:e20216466. https://doi.org/10.17665/1676-4285.20216466

7. Rodríguez-Palma M, Verdú-Soriano J, Soldevilla-Agreda JJ, Pancorbo-Hidalgo PL, García-Fernández FP. Conceptual Framework for Incontinence - Associated Dermatitis Based on Scoping Review and Expert Consensus Process. J Wound Ostomy Continence Nurs. 202;48(3): 239-50. https://doi.org/10.1097/WON.0000000000000754

8. Moraes JT, Borges EL, Lisboa CR, Cordeiro DCO, Rosa EG, Rocha NA. Conceito e classificação de lesão por pressão: atualização no National Pressure Ulcer Advisory Panel. Enferm Cent O Min. 2016;6(2): 2292-2306. https://doi.org/10.19175/recom.v6i2.1423

9. Alves LAF, Santana RF, Cardozo AS, Souza TM, Silva CFR. Dermatitis Associated with Incontinence and the Not-Standard Use of Geriatric Diaper: SystematicReview. ESTIMA Braz J Enterestomal Ther. 2016; 14(4):203-13. https://doi.org/10.5327/Z1806-3144201600040007

10. Zangirolami-Raimundo J, Echeimberg JO, Leone C. Tópicos de metodologia de pesquisa: Estudos de corte transversal. Journal of Human Growth and Development. 2018;28(3):356-60. https://doi.org/10.7322/jhgd.152198 
11. Polit DF, Beck CT, Hungler BP. Fundamentos de pesquisa em enfermagem: avaliação de evidência para a prática da enfermagem. $7^{\mathrm{a}}$ ed. São Paulo: Artmed; 2011.

12. Folstein MF, Folstein SE, McHugh PR. Mini-mental state: a practical method for grading the cognitive state of patients for the clinician. J Psychiatr Res. 1975;12(13):189-98. https://doi.org/10.1016/0022-3956(75)90026-6

13. Costa TNM, Nieto JPS, Morikawa LS, Araújo AVS, Cardoso AAM, Mafra BG et al. Analysis of Folstein's Mini State examination in institutionalized and non institutionalized elderly people. Brazilian Journal of Health Review. 2021;4(2):8319-36. https://doi. org/10.34119/bjhrv4n2-357

14. Tamanini JTN, Dambros M, D'Ancona CAL, Palma PCR, Rodrigues Netto Junior N. Validation of the "International Consultation on Incontinence Questionnaire - Short Form" (ICIQ-SF) for Portuguese. Rev Saúde Pública. 2004;38(3):438-44. https://doi. org/10.1590/S0034-89102004000300015

15. Timmermans $L$, Falez F, Mélot $C$, Wespes $E$. Validation of use of the international consultation on incontinence questionnaireurinary incontinence-short form (ICIQ-UI-SF) for impairment rating: A transversal retrospective study of 120 patients. Neurourology and Urodynamics 2013;32(7):974-79. https://doi.org/10.1002/nau.22363

16. Yates A. Incontinence-associated dermatitis 1: riskfactors for skindamage. Nursing Times. 2020;116(3):46-50. Disponível em: https://www.nursingtimes.net/clinical-archive/tissue-viability/incontinence-associated-dermatitis-1-risk-factors-for-skindamage-02-03-2020/

17. Werth SL, Justice R. Prevalence of Moisture-Associated Skin Damage in an Acute Care Setting: Outcomes From a Quality Improvement Project. J Wound Ostomy Continence Nurs. 2019;46(1):51-4. https://doi.org/10.1097/WON.0000000000000499

18. Lopes EB, Pupulim JSL, Oliva APV. Perfil dos diagnósticos de enfermagem de pacientes internados em unidade de clínica médica. Cienc Cuid Saude. 2016;15(2):358-65. https://doi.org/10.4025/cienccuidsaude.v15i2.29356

19. Nobre IEAM, Barros LM, Gomes MLS, Silva LA, Lima ICS, Caetano JÁ. Sistema de classificação de pacientes de FUGULIN: perfil assistencial da clínica médica. Rev enferm UFPE online. 2017;11(4):1736-42. https://doi.org/10.5205/1981-8963v11i4a15245p1736-1742-2017

20. Grden CRB, Martins AR, Cabral LPA, Reche PM, Arcaro G, Brasil D et al. Incontinence associated dermatitis in elderly people admitted to a university hospital. Rev Bras Enferm. 2020;73(Suppl 3):e20190374. https://doi.org/10.1590/0034-7167-2019-0374

21. Malta DC, Bernal RTI, Lima MG, Araújo SSC, Silva MMA, Freitas MIF et al. Doenças crônicas não transmissíveis e a utilização de serviços de saúde: análise da Pesquisa Nacional de Saúde no Brasil. Rev Saude Publica. 2017; 51(Supl 1):4s. https://doi. org/10.1590/S1518-8787.2017051000090

22. Bitencourt GR, Alves LAF, Santana RF. Practice of use of diapers in hospitalized adults and elderly: cross-sectional study. Rev Bras Enferm. 2018;71(2):343-9. http://doi.org/10.1590/0034-7167-2016-0341 